\title{
Preferences for end-of-life care for children with cancer
}

\author{
Caprice Knapp PhD, Kelly Komatz MD
}

See related research article by Tomlinson and colleagues at www.cmaj.ca/lookup/doi/10.1503/cmaj.110392

Competing interests: None declared.

This article was solicited and has not been peer reviewed.

Correspondence to: Dr. Caprice Knapp, cak@ichp.ufl.edu

CMAJ 2011. DOI:10.1503 /cmaj.111557

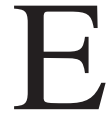
very time cancer is diagnosed in a child, a journey begins. For many, advances in diagnostics and treatment allow the journey to end with a cure. The National Cancer Institute estimates that of the 10 million cancer survivors in the United States today, about 270000 received their diagnoses as children. ${ }^{1}$

Although survivorship rates vary by site and stage of disease, about $79.6 \%$ of children with newly diagnosed cancer will survive after five years. ${ }^{2}$ However, there are a small number of children who will not survive. Their transition through the cancer continuum ultimately leads to the end-of-life stage where they and their families are faced with myriad medical and ethical decisions. One such decision is the choice between palliative chemotherapy and supportive care. The goal of palliative chemotherapy is to slow the growth of the cancer. However, chemotherapy is invasive, requiring monitoring in laboratories and potentially causing negative side effects and symptoms. Alternatively, palliative supportive care is meant not to cure, but to improve the quality of life.

The decision whether to undergo palliative chemotherapy or supportive care is particularly complex among pediatric patients. Parents, being the legal authority for medical decision-making on behalf of their children, must gather information, negotiate and serve as the final decisionmakers. Parents grapple with the realization that although supportive care may improve or maintain their child's quality of life, it may not affect the length of that life. Moreover, children want to be heard. Studies have overwhelmingly found that children, especially adolescents, want to participate in making medical decisions. ${ }^{3,4}$ Children also want to please their parents, which may

\section{KEY POINTS}

- Little is known about the decision-making preferences surrounding end-of-life care for children.

- Parents and health care workers hold different opinions regarding when, if ever, treatment should end and supportive care should begin.

- Incongruity between preferences could be attributed to the importance of hope in parental decision-making. cause conflict if their treatment preferences differ. In addition, health care workers play an important role in end-of-life decisions. They must provide information, explain benefits and risks, estimate survival and perhaps voice preferences, all in a culturally competent and ethical manner.

Little is known about decision-making preferences surrounding end-of-life care for children. Most studies that have attempted to gauge preferences have focused on the experiences of the parents. For example, a 2010 study looked at the frequency of decisional conflict and its associated factors among 140 parents of children with life-limiting illnesses. ${ }^{5}$ The authors found that parents with less than a high school education had greater decisional conflict than parents who had attended at least some college. Another study involving 43 parents suggested that parents fear disagreeing with health care workers over medical decisions concerning end-of-life care for their children. ${ }^{6}$

In their recent study published in CMAJ, Tomlinson and colleagues add to the scant information available on end-of-life decision-making for children and pediatric palliative care. ${ }^{7}$ The purpose of their study was to compare preferences for end-of-life treatment between health care workers and parents of children who were not expected to survive. A total of 77 parents and 128 health care workers participated in the fouryear study. Visual aids and prepared scripts, which were extensively tested by a focus group before the study began, were used during the interviews. The results suggest that parents favour palliative chemotherapy, whereas health care workers prefer supportive care. Tomlinson and colleagues also found that parents rank hope as an important factor in their decision-making.

This study is important because it highlights the incongruity between the preferences of parents and those of health care workers. However, it may be that this incongruity masks a greater concern: miscommunication or unrealistic expectations. The Center to Advance Palliative Care recently published research on public opinion on palliative care. ${ }^{8} \mathrm{~A}$ key finding in the report was that most physicians do not understand the scope 
of palliative care. Therefore, if there is a basic misunderstanding of terminology, definitions and messages associated with end-of-life care, then incongruity of preferences might be expected.

Another important finding in the study by Tomlinson and colleagues is the importance of hope. A 2010 study of 25 parents whose children died from brain tumours found that parents use hope as a coping mechanism. ${ }^{9}$ It is therefore difficult to balance hope for a cure with hope for the comfort and dignity of the child.

Finally, the study by Tomlinson and colleagues ${ }^{7}$ is important because of its design and rigor. The study team pilot-tested the visual aids and interview guide with 12 parents whose children had died from cancer. Participatory and action research is critical in palliative care. Although researchers and health care workers can leverage their experience when designing tools for collecting data, the opinions of families that have lost a child must be considered. In addition, researchers must be prepared for emotional reactions and have a plan to address them. For this reason, the involvement of clinicians trained to treat psychosocial issues (such as child psychologists, social workers and chaplains) is crucial. Furthermore, evidence in pediatric palliative care is limited by small sample sizes. Tomlinson and colleagues are to be commended for consistently doing their study over four years. ${ }^{7}$ Such an endeavour takes commitment from everyone involved. Compared with many populations, 77 completed interviews may seem small; however, for researchers in this field, it is an impressive number that should be acknowledged.

Of course, no study is without its limitations. The authors acknowledge two important drawbacks to their research. First, children's preferences were not obtained. Not collecting children's preferences fails to recognize that decision-making is triadic, not dyadic. Without this information, it is unclear whether families or health care workers drive incongruity. Second, most of the participating health care professionals were nurses, who typically do not make referrals or write orders for chemotherapy.
Despite these limitations, Tomlinson and colleagues have conducted an impressive study. ${ }^{7}$ Extensions of this work are warranted. Future studies might explore how these results differ for programs where curative and supportive teams are integrated. In such programs, multidisciplinary teams of health care professionals work together with families to address both active treatment and support. When families become familiar with both concepts, the eventual transition should be easier for parents to accept. The framework set forth by Tomlinson and colleagues ${ }^{7}$ could be used in such a groundbreaking study.

\section{References}

1. Ries LAG, Smith MA, Gurney JG, et al., editors. Cancer incidence and survival among children and adolescents: United States SEER Program 1975-1995. Bethesda (MD): National Cancer Institute; 1999.

2. National Cancer Institute Fact Sheet: Childhood cancers Bethesda (MD): National Cancer Institute. Available: www.cancer .gov/cancertopics/factsheet/Sites-Types/childhood (accessed 2011 Sept. 13).

3. Taylor S, Haase-Casanovas S, Weaver T, et al. Child incolvement in the paediatric consultation: a qualitative study of children and carers' views. Child Care Health Dev 2010;36:678-85.

4. Zwaanswijk M, van Dulmen S, Hoogerbrugge PM, et al. Communicating with child patients in pediatric oncology consultations: a vignette study on child patients', parent's, and survivors' communication preferences. Psychooncology 2011;20:269-77.

5. Knapp C, Madden V, Wang H, et al. Factors affecting decisional conflict for parents with children enrolled in a paediatric palliative care programme. Int J Palliat Nurs 2010;16:542-7.

6. Hinds PS, Oakes L, Quargnenti A, et al. An international feasibility study of parental decision making in pediatric oncology. Oncol Nurs Forum 2000;27:1233-43.

7. Tomlinson D, Bartels U, Gammon J, et al. Chemotherapy versus supportive care alone in pediatric palliative care for cancer: comparing the preferences of parents and health care professionals. CMAJ 2011;183:E1252-58

8. Center to Advance Palliative Care. 2011 public opinion research on palliative care: a report based on research by public opinion strategies. New York (NY): The Center; 2011. Available: www.capc.org/tools-for-palliative-care-programs/marketing/public -opnion-research/2011-public-opinion-research-on-palliative-care .pdf (accessed 2011 Sept. 13).

9. Zelcer S, Cataudella D, Calmey AE, et al. Palliative care of children with brain tumors: a parental perspective. Arch Pediatr Adolesc Med 2010;164:225-30.

Affiliations: Caprice Knapp is with the Department of Epidemiology, and Kelly Komatz is with the Department of Pediatrics, University of Florida, Gainesville, Fla.

Contributors: Both authors made substantial contributions to the conception and design of the article, drafted the article and revised it critically for important intellectual content and approved the final version submitted for publication. 